\title{
Influence of Integrated Weed Management Practices on Dynamics and Weed Control Efficiency in Dry Direct Seeded Rice
}

\author{
K.P. Bhurer ${ }^{1}$, D.N. Yadav ${ }^{2}$, J.K. Ladha ${ }^{3}$, R.B. Thapa ${ }^{2}$ and K.R. Pandey ${ }^{2}$ \\ ${ }^{1}$ Nepal Agriculture Research Council(NARC), Lalitpur \\ ${ }^{2}$ Institute of Agriculture and Animal Sciences (IAAS),Rampur Chitwan \\ ${ }^{3}$ IRRI, Los Banos, Philippines \\ e-mail:Kailash2092@yahoo.com
}

\begin{abstract}
Field experiment under dry direct seeded rice(Oryza sativa L.) was conducted during rainy season of 2010 and 2011 at Regional Agriculture Research Station (RARS), Parwanipur, Bara to develop appropriate weed management practices for dry direct- seeded rice. The trial was laid-out in randomized complete block design (RCBD) and replicated thrice. Observations were taken on weed, plant growth and yield attributing, yield, and socio-economic parameters. The weed density, dry weed weight and weed control efficiency resulted significantly different as influenced by integrated weed management practices. Low weed population density, low weed index and highest weed control efficiency resulted by pendimethalin followed by 2, 4- D followed by one hand weeding were at par with weed free check. Highest yield resulted from weed free plot followed by pendimethalin followed by two hand weeding and pendimethalin followed by 2, 4- D followed by one hand weeding. However, the net return per unit investment resulted highest in pendimethalin followed by 2, 4- $\mathrm{D}$ followed by one hand weeding. This proved that amid increasing wage rate and labour scarcity integrated weed management through pendimethalin 30 EC (Stomp) @1 kg a. i. /ha as pre- emergence herbicide application followed by 2, 4- D sodium salt 80 WP @ $0.5 \mathrm{~kg}$ a. i. /ha followed by one hand weeding or stale seed bed followed by pendimethalin 30 EC (Stomp) @1 kg a. i. /ha followed by bispyribac (Nominee gold) @25 g a. i. /ha 10\% @200 ml/ha at 20 days of seeding resulted best alternative for manual hand weeding practices giving higher net return per unit investment.
\end{abstract}

Key words: dry direct seeded rice, herbicide, 2,4-D, pendimethaliny and weed index

\section{Introduction}

Rice (Oryza sativa L.) is the main staple food of Nepalese people -which has_occupied the_first position in terms of area coverage (1,531,493 ha) and production (5072248 mt) whereas average productivity is $3.31 \mathrm{t} /$ ha, which is slightly in increasing trends (MoAD 2011/ 12) but the cost of cultivation is becoming high during these days due to increment in labor wages beside unavailability of labor in peak period of operation. Puddled transplanting is the dominant method of rice establishment in Asia including Nepal which provides multiple benefits to rice including reduction in weed population and percolation losses and increases availability of nutrients (Sanches 1973). However, it deteriorates soil physical properties ultimate adversely affects the growth and productivity of succeeding wheat crop. All these factors have increased the interest of farmers to shift from the conventional practice of puddled transplanting (CT- TPR) to direct seeded rice (DSR), especially dry DSR.

Direct seeded rice (DSR) is a cost effective rice establishment method where dry seed is drilled into the non-puddled soil. This provides opportunities of saving irrigation water by $12-35 \%$, labour up to $60 \%$ 
and provides higher net returns (US\$30-50 ha ${ }^{-1}$ ) with similar or slightly lower yield of rice (Kumar and Ladha 2011). Despite multiple benefits of dry- DSR, weed control remains one of the major challenges for its success in South Asia (Kumar and Ladha, 2011: Rao et al. 2007, Singh et al., 2008). Weed control is more difficult in dry- DSR than CT- TPR because of simultaneously emerging rice seedlings with weeds in dry - DSR which are less competitive than 30-35 days old rice seedlings use in CT- TPR and initial flooding used in CT- TPR is effective for weed control but it is lacking in dry- DSR (Kumar and Ladha 2011, Rao et al. 2007).

Thus, weeds are the most severe constraint to aerobic rice production and timely weed management is crucial for increasing the productivity of aerobic rice. In Nepal, Ranjit (2007) observed that weeds caused yield loss in direct seeded rice ranging from 14-93 \% where as in transplanted rice it was 17- $47 \%$. Most upland and aerobic rice growers in Asia mechanically weed their crops two or three times per season, investing up_to 190 person days ha-1 in hand weeding (Roder 2001). Herbicides are considered to be an alternative/ supplement to hand weeding. Both Pre and Postemergence herbicides can be used in aerobic rice fields, which are effective, if properly used (De Datta \& Baltzar 1996, Singh et al. 2006).

For achieving effective weed control in dry- DSR, it is important to develop integrated weed management (IWM) program comprising of chemical, physical, cultural and biological methods built on a foundation of good knowledge of weed biology and ecology. The effective weed management practices include stale bed technique, crop rotation, zero tillage ZT, use of competitive varieties, water management, mulching, intercropping or cover crops and use of suitable chemicals at the right time.

The impact of the spread of direct seeding has been positive over all. DSR reduces the climatic risk caused by unpredictable monsoon rains, reducing dependence on pump sets and tractors for timely crop establishment which will benefit poor farmers. The use of direct seeding increases the opportunity for farmers to plant a winter crop on residual moisture. It also advances harvest by 18 days. Harvesting earlier reduces the risk of terminal drought at heading to maturity stage in rice at time of monsoon end which also abruptly increases opportunity for establishing post _rice winter crop. However, this type of research is less carried in our context. Therefore, the present experiment was conducted to develop effective and economical integrated weed management practices for dry direct-seeded rice.

\section{Methodology}

The experiment was conducted at the Regional Agricultural Research Station (RARS), Parwanipur, Bara during the rainy season of 2010 and 2011_in randomized block design replicated thrice. The site is located in the central piedmont of Nepal at 115 masl. at $27^{\circ} 21^{\prime} \mathrm{N}$ and $84^{\circ} 53^{\prime} \mathrm{E}$. The soil is an ifnceptisol formed on Himalayan residuum with the following characteristics in the top 15 -cm profile: clay $8.0 \%$, slit $17.0 \%$, sand $75.0 \%$ (loamy sand), $\mathrm{pH}$ (1:2 soil:water) 7.0 , total $\mathrm{N} 0.86 \mathrm{~g} \mathrm{~kg}^{-1}$, total C $6.5 \mathrm{~g} \mathrm{~kg}^{-1}, \mathrm{NH}_{4} \mathrm{OAc}-$ extractable $\mathrm{K} 0.054 \mathrm{~g} \mathrm{~kg}^{-1}$, Olsen p $0.015 \mathrm{~g} \mathrm{~kg}^{-1}$, saturation extract exchangeable cations $1.4 \mathrm{dS} \mathrm{m}^{-1}$, and bulk density $1.6 \mathrm{Mg} \mathrm{m}^{-3}$.

Ten different weed management practices were tested against weedy and weed free check. For stale seed bed glyphosate 41\%@1kg a.i/ha and paraquat @ 0.5 $\mathrm{kg}$ a.i/ha were applied before one week of seeding. Pendimethalin 30 EC (Stomp) @ 1 kg a.i/ha were as a pre-emergence herbicide in moist condition at evening hours in five treatments. Mulching (wheat straw) @ $4.0 \mathrm{t} / \mathrm{ha}$ was done on the next day. Bispyribac sodium (Nominee gold) 10\% @25 g a.i/ha @ 200ml/ha was applied at 20 days of seeding as post-emergence. 2, 4D sodium salt (80 WP) was applied @ $0.5 \mathrm{~kg}$ a.i $/$ ha at 30 days after seeding to kill Sesbania. Continuous weeding was done to weed free treatment to clear field at weekly interval in weed free treatment, and hand weeding was done at 20 days after sowing (DAS) in hand weeding treatment. The trial was laid out in randomized block design with three replications.

Sabitri cv of rice was sown on $7^{\text {th }}$ July 2010, $15^{\text {th }}$ June 2011_at the rate of $30 \mathrm{~kg} / \mathrm{ha}$ with Pantnagar Zero Till Drill in line. Row to row spacing was kept $20 \mathrm{~cm}$ and plant to plant spacing was made continuous. Seeds of Sesbania aculeata were also sown at the rate of 15 $\mathrm{kg} /$ ha for brown manuring (compost of straw and other remaining in the field) in mentioned plots. Nitrogen, phosphorous and potash fertilizers were applied @ 100: $50: 30 \mathrm{~kg} / \mathrm{ha}$ whereas, nitrogen applied through urea $(46 \% \mathrm{~N})$ and phosphorus through DAP $(18 \% \mathrm{~N}$ and $46 \% \mathrm{P}_{2} \mathrm{O}_{5}$ ) and potash through muriate of potash $\left(60 \% \mathrm{~K}_{2} \mathrm{O}\right)$. Half dose of nitrogen, full dose of 
K.P. Bhurer et al./Influence of Integrated Weed.....

phosphorous and potash were applied as basal dose and remaining half of nitrogen was applied in two split doses first at active tillering and second at panicle initiation stages in all the treatments. Pre-sowing irrigation was provided before stale-seedbed. Irrigation was applied in the field as per requirement. About $5 \mathrm{~cm}$ water was maintained either through deep tube well or by pumping set regularly up to grain filling stage of rice crop. chlorpyriphos (Dursban ) @ 2 ml/litre of water was applied before milking stage of the crop to-protect it sucking from gundhi bug (Leptocorisa varicornis).
Observations were taken on weed density, dry weed weight, weed index and weed control efficiency at 30 and 60 days after sowing from one meter square area. Plants were harvested from the net plot area $\left(15 \mathrm{~m}^{2}\right)$ and left in the field for 5-7 days for sun drying. Threshing was done on cemented threshing floor manually and the after grains were cleaned by winnowing. The grain yield was recorded at $12 \%$ moisture. The grain yield, straw yield, grain straw The index were recorded from the net plot of $15 \mathrm{~m}^{2}$ areas. Final data were analyzed by standard statistical techniques (MSTAT- C package).

Table 1. Weed density at 30 DAS as influenced by integrated weed management practices in dry direct seeded rice at RARS, Parwanipur, Bara

\begin{tabular}{|c|c|c|c|c|c|c|c|}
\hline \multirow{3}{*}{ SN } & \multirow[b]{2}{*}{ Treatments } & \multicolumn{6}{|c|}{ Weed density $($ no/m²) $30 \mathrm{DAS}$} \\
\hline & & \multicolumn{2}{|c|}{ Broad leaves } & \multicolumn{2}{|c|}{ Sedges } & \multicolumn{2}{|c|}{ Grasses } \\
\hline & & 2010 & 2011 & 2010 & 2011 & 2010 & 2011 \\
\hline 1 & Weedy & $\begin{array}{l}112.40 \mathrm{~A} \\
(10.22 \mathrm{~A})\end{array}$ & $\begin{array}{l}15.10 \mathrm{~B} \\
(3.94 \mathrm{~A})\end{array}$ & $\begin{array}{l}40.60 \mathrm{~B} \\
(6.41 \mathrm{~B})\end{array}$ & $\begin{array}{c}12.60 \mathrm{AB} \\
(3.61 \mathrm{~A})\end{array}$ & $\begin{array}{l}104.40 \mathrm{~A} \\
(10.15 \mathrm{~A})\end{array}$ & $\begin{array}{l}12.76 \mathrm{~B} \\
(3.64 \mathrm{~A})\end{array}$ \\
\hline 2 & Weed free & $0.00 \mathrm{C}(0.71 \mathrm{D})$ & $\begin{array}{c}0.00 \mathrm{~B} \\
(0.71 \mathrm{~F})\end{array}$ & $\begin{array}{c}0.00 \mathrm{E} \\
(0.71 \mathrm{H})\end{array}$ & $\begin{array}{l}0.00 \mathrm{AB} \\
(0.71 \mathrm{~F})\end{array}$ & $0.00 \mathrm{C}(0.71 \mathrm{~F})$ & $\begin{array}{c}0.00 \mathrm{~B} \\
(0.71 \mathrm{~F})\end{array}$ \\
\hline 3 & Pendime thalin to bispynbac & $68.60 \mathrm{~B}(798 \mathrm{~B})$ & $\begin{array}{l}11.45 \mathrm{C} \\
(3.45 \mathrm{~B})\end{array}$ & $\begin{array}{l}146.90 \mathrm{~A} \\
(12.14 \mathrm{~A})\end{array}$ & $\begin{array}{c}9.08 \mathrm{C} \\
(3.09 \mathrm{~B})\end{array}$ & $\begin{array}{l}62.00 \mathrm{~B} \\
(7.90 \mathrm{~B})\end{array}$ & $\begin{array}{c}11.68 \mathrm{D} \\
(3.48 \mathrm{AB})\end{array}$ \\
\hline 4 & $\begin{array}{l}\text { Pendirre thalin to two hand } \\
\text { weeding }\end{array}$ & $\begin{array}{c}7.40 \mathrm{C} \\
(2.51 \mathrm{CD})\end{array}$ & $\begin{array}{c}0.50 \mathrm{C} \\
(0.99 \mathrm{E})\end{array}$ & $\begin{array}{c}1.30 \mathrm{DE} \\
(1.33 \mathrm{FG})\end{array}$ & $\begin{array}{l}0.43 \mathrm{~B} \\
(0.96 \mathrm{~F})\end{array}$ & $4.80 \mathrm{C}(2.30 \mathrm{E})$ & $\begin{array}{c}3.38 \mathrm{C} \\
(1.97 \mathrm{D})\end{array}$ \\
\hline 5 & Stale seedbed fb bispynbac & $8.90 \mathrm{C}(280 \mathrm{C})$ & $\begin{array}{c}1.08 \mathrm{~B} \\
(1.24 \mathrm{D})\end{array}$ & $\begin{array}{l}4.50 \mathrm{DE} \\
(2.23 \mathrm{E})\end{array}$ & $\begin{array}{l}3.83 \mathrm{AB} \\
(2.07 \mathrm{D})\end{array}$ & $\begin{array}{r}10.80 \mathrm{C} \\
(3.35 \mathrm{D})\end{array}$ & $\begin{array}{c}6.88 \mathrm{~B} \\
(2.71 \mathrm{C})\end{array}$ \\
\hline 6 & $\begin{array}{l}\text { Stale seedbed fo } \\
\text { Pendime thalin to bispynbac }\end{array}$ & $\begin{array}{c}8.20 \mathrm{C} \\
(2.49 \mathrm{CD})\end{array}$ & $\begin{array}{c}0.95 \mathrm{~B} \\
(1.20 \mathrm{DE})\end{array}$ & $\begin{array}{l}1.50 \mathrm{DE} \\
(1.41 \mathrm{~F})\end{array}$ & $\begin{array}{l}3.40 \mathrm{AB} \\
(1.97 \mathrm{D})\end{array}$ & $\begin{array}{c}9.70 \mathrm{C} \\
(3.19 \mathrm{DE})\end{array}$ & $\begin{array}{l}4.43 \mathrm{~B} \\
(2.22 \mathrm{D})\end{array}$ \\
\hline 7 & $\begin{array}{l}\text { Mulch 4t/ha fo bispynbac to } \\
\text { One hand weedirg }\end{array}$ & $13.10 \mathrm{C}(334 \mathrm{C})$ & $\begin{array}{c}8.03 \mathrm{C} \\
(2.92 \mathrm{C})\end{array}$ & $\begin{array}{c}6.80 \mathrm{D} \\
(2.68 \mathrm{D})\end{array}$ & $\begin{array}{l}5.48 \mathrm{C} \\
(2.44 \mathrm{C})\end{array}$ & $\begin{array}{l}18.80 \mathrm{C} \\
(4.39 \mathrm{C})\end{array}$ & $\begin{array}{l}10.28 \mathrm{DE} \\
(3.27 \mathrm{~B})\end{array}$ \\
\hline 8 & $\begin{array}{l}\text { Stale seedbed fo mulch } 4 \text { t'ha fb } \\
\text { bispynbac } \\
\text { Pendime thalin and Sesbania }\end{array}$ & $56.90 \mathrm{~B}(7.13 \mathrm{~B})$ & $\begin{array}{c}0.78 \mathrm{C} \\
(1.13 \mathrm{DE})\end{array}$ & $\begin{array}{l}13.60 \mathrm{C} \\
(3.75 \mathrm{C})\end{array}$ & $\begin{array}{l}1.64 \mathrm{AB} \\
(1.45 \mathrm{E})\end{array}$ & $\begin{array}{l}53.10 \mathrm{~B} \\
(7.29 \mathrm{~B})\end{array}$ & $\begin{array}{c}3.65 \mathrm{~B} \\
(2.04 \mathrm{D})\end{array}$ \\
\hline 9 & $\begin{array}{l}\text { co-culture fb } 2,4 \mathrm{D} \text { Na salt fb } \\
\text { one hand weedirg }\end{array}$ & $63.60 \mathrm{~B}(7.41 \mathrm{~B})$ & $\begin{array}{l}11.05 \mathrm{~A} \\
(3.39 \mathrm{~B})\end{array}$ & $\begin{array}{l}15.30 \mathrm{C} \\
(3.96 \mathrm{C})\end{array}$ & $\begin{array}{c}8.10 \mathrm{~A} \\
(2.92 \mathrm{~B})\end{array}$ & $\begin{array}{l}65.10 \mathrm{~B} \\
(8.08 \mathrm{~B})\end{array}$ & $\begin{array}{c}11.78 \mathrm{~A} \\
(3.50 \mathrm{AB})\end{array}$ \\
\hline 10 & $\begin{array}{l}\text { Pendime thalin to } 2,4 \mathrm{D} \text { to one } \\
\text { hand weeding }\end{array}$ & $\begin{array}{c}5.74 \mathrm{C} \\
(2.32 \mathrm{CD})\end{array}$ & $\begin{array}{l}0.45 \mathrm{C} \\
0.97 \mathrm{E})\end{array}$ & $\begin{array}{c}0.50 \mathrm{E} \\
(1.00 \mathrm{GH} \\
)\end{array}$ & $\begin{array}{c}0.30 \mathrm{C} \\
(0.88 \mathrm{~F})\end{array}$ & $4.60 \mathrm{C}(2.26 \mathrm{E})$ & $\begin{array}{c}1.88 \mathrm{E} \\
(1.53 \mathrm{E})\end{array}$ \\
\hline & Mean & $34.48(4.69)$ & $4.94(1.99)$ & $\begin{array}{c}23.10 \\
(12.14)\end{array}$ & $\begin{array}{c}4.48 \\
(2.01)\end{array}$ & $33.33(4.96)$ & $6.67(2.50)$ \\
\hline & $\mathrm{CV} \%$ & $64.61(26.47)$ & $\begin{array}{l}18.22 \\
(7.81)\end{array}$ & $\begin{array}{l}15.61 \\
(3.56)\end{array}$ & $\begin{array}{l}22.69 \\
(9.85)\end{array}$ & $36.29(12.78)$ & $\begin{array}{l}17.27 \\
(7.58)\end{array}$ \\
\hline & F-test & $* *$ & $* * *$ & $* *$ & $* *$ & $* *$ & $* *$ \\
\hline & LSD at 0.05 & 32.33 & 1.306 & 5.232 & 1.476 & 17.55 & 1.67 \\
\hline
\end{tabular}

\section{Results and Discussion}

Effect on weed density and dry weed weight

The important weeds infesting in the cropped area were Cynodon doctylon, Cyperus rotundus, C. iria,
Echinochloa crusgalli, E. colonum, Fimbristylis dichotoma, Phyllanthus niruri etc. Data pertaining to weed density as influenced by integrated weed management practices in its dry direct seeded rice are 
presented in Table 1 and Table 2. Irrespective of the years, weed control methods significantly influenced the weed population and dry matter production. There were remarkable variation in weed density and dry weed weight between two years Weed density and weed dry weight were observed higher in 2010 as compared to 2011. The crop experienced severe weed competition in 2010 which might be due to favorable weather condition leading to vigorous weed growth. Behera and Jena (1998) found similar result in DSR. Significantly highest weed population and biomass were found when the weeds were not disturbed during the whole season of crop growth (weedy check i.e. control) while the lowest weed population was observed in weed free treatment. All weed control treatments significantly reduced density and dry weight of weed over weedy check in both years. Among the herbicidal treatments, maximum weed density and biomass were recorded in Pendimethalin followed by Bispyribac and the minimum in pendimethalin followed by 2, 4- $\mathrm{D}_{2}$ followed by one hand weeding. It was closely followed by Pendimethalin, followed by two hand weeding. Detail results are presented in Table-1, Table $=2$, Table $=3$ and Table $=4$.

Table 2. Weed density at 60 DAS as influenced by integrated weed management practices in dry direct seeded rice at Parwanipur, Bara

\begin{tabular}{|c|c|c|c|c|c|c|c|}
\hline \multirow{3}{*}{$5 \mathrm{~N}$} & \multirow{3}{*}{ Treatments } & \multicolumn{6}{|c|}{ Weed density $\left(\mathrm{no} / \mathrm{m}^{2}\right)$ at 60 DAS } \\
\hline & & \multicolumn{2}{|c|}{ Broad leaves } & \multicolumn{2}{|c|}{ Sedges } & \multicolumn{2}{|c|}{ Grasses } \\
\hline & & 2010 & 2011 & 2010 & 2011 & 2010 & 2011 \\
\hline 1 & Weedy & $\begin{array}{l}46.70 \mathrm{~A} \\
(6.74 \mathrm{~A})\end{array}$ & $\begin{array}{c}12.18 \mathrm{ABC} \\
(3.56 \mathrm{~A})\end{array}$ & $\begin{array}{l}16.50 \mathrm{~A} \\
(4.11 \mathrm{~A})\end{array}$ & $\begin{array}{l}21.80 \mathrm{AB} \\
(4.72 \mathrm{~A})\end{array}$ & $\begin{array}{l}51.00 \mathrm{~A} \\
(7.17 \mathrm{~A})\end{array}$ & $\begin{array}{l}18.93 \mathrm{~B} \\
(4.41 \mathrm{~A})\end{array}$ \\
\hline 2 & Weed free & $\begin{array}{l}0.00 \mathrm{~B} \\
(0.71 \mathrm{C})\end{array}$ & $\begin{array}{l}0.00 \mathrm{BC} \\
(0.71 \mathrm{D})\end{array}$ & $\begin{array}{c}0.00 \mathrm{E} \\
(0.71 \mathrm{G})\end{array}$ & $\begin{array}{c}0.00 \mathrm{~B} \\
(0.71 \mathrm{G})\end{array}$ & $\begin{array}{c}0.00 \mathrm{~F} \\
(0.71 \mathrm{G})\end{array}$ & $\begin{array}{l}0.00 \mathrm{~B} \\
(0.71 \mathrm{~F})\end{array}$ \\
\hline 3 & Pendimethalin fb bispynbac & $\begin{array}{l}44.20 \mathrm{~A} \\
(6.64 \mathrm{~A})\end{array}$ & $\begin{array}{l}11.08 \mathrm{~A} \\
(3.39 \mathrm{~A})\end{array}$ & $\begin{array}{l}12.10 \mathrm{~B} \\
(3.53 \mathrm{~B})\end{array}$ & $\begin{array}{c}16.80 \mathrm{~A} \\
(4.12 \mathrm{BC})\end{array}$ & $\begin{array}{l}32.80 \mathrm{C} \\
(5.76 \mathrm{~B})\end{array}$ & $\begin{array}{l}16.50 \mathrm{~A} \\
(4.12 \mathrm{~A})\end{array}$ \\
\hline 4 & Pendimethalin fo two hand weeding & $\begin{array}{c}2.80 \mathrm{~B} \\
(1.70 \mathrm{BC})\end{array}$ & $\begin{array}{c}5.05 \mathrm{ABC} \\
(2.35 \mathrm{C})\end{array}$ & $\begin{array}{c}0.80 \mathrm{DE} \\
(1.11 \mathrm{EFG})\end{array}$ & $\begin{array}{l}9.90 \mathrm{AB} \\
(3.21 \mathrm{E})\end{array}$ & $\begin{array}{c}1.11 \mathrm{~F} \\
(1.27 \mathrm{~F})\end{array}$ & $\begin{array}{l}3.55 \mathrm{~B} \\
(2.01 \mathrm{E})\end{array}$ \\
\hline 5 & Stale seedbed fb bispyribac & $\begin{array}{c}3.80 \mathrm{~B} \\
(2.03 \mathrm{BC})\end{array}$ & $\begin{array}{l}10.13 \mathrm{ABC} \\
(3.25 \mathrm{AB})\end{array}$ & $\begin{array}{c}1.80 \mathrm{DE} \\
(151 \mathrm{DE})\end{array}$ & $\begin{array}{c}15.30 \mathrm{~B} \\
(3.97 \mathrm{BC})\end{array}$ & $\begin{array}{c}4.60 \mathrm{~F} \\
(2.22 \mathrm{E})\end{array}$ & $\begin{array}{l}9.93 \mathrm{~B} \\
(3.22 \mathrm{C})\end{array}$ \\
\hline 6 & $\begin{array}{l}\text { Stale seedbed fb Pendimethalin fb } \\
\text { bispynbac }\end{array}$ & $\begin{array}{c}3.10 \mathrm{~B} \\
(1.81 \mathrm{BC})\end{array}$ & $\begin{array}{c}8.60 \mathrm{C} \\
(3.01 \mathrm{~B})\end{array}$ & $\begin{array}{c}1.10 \mathrm{DE} \\
(1.26 \mathrm{EF})\end{array}$ & $\begin{array}{c}14.20 \mathrm{~B} \\
(3.8 \mathrm{CD})\end{array}$ & $\begin{array}{c}1.90 \mathrm{~F} \\
(1.53 \mathrm{~F})\end{array}$ & $\begin{array}{l}9.23 \mathrm{~B} \\
(3.11 \mathrm{C})\end{array}$ \\
\hline 7 & $\begin{array}{l}\text { Mulch } 4 \text { t'ka fb bispynibac to One hand } \\
\text { weeding }\end{array}$ & $\begin{array}{l}8.00 \mathrm{~B} \\
(2.85 \mathrm{~B})\end{array}$ & $\begin{array}{c}10.40 \mathrm{E} \\
(3.30 \mathrm{AB})\end{array}$ & $\begin{array}{l}3.10 \mathrm{D} \\
(1.87 \mathrm{D})\end{array}$ & $\begin{array}{c}16.38 \mathrm{D} \\
(4.10 \mathrm{BC})\end{array}$ & $\begin{array}{c}9.30 \mathrm{E} \\
(3.12 \mathrm{D})\end{array}$ & $\begin{array}{l}12.40 \mathrm{D} \\
(3.59 \mathrm{~B})\end{array}$ \\
\hline 8 & $\begin{array}{l}\text { Stale seedbed fo mulchutt/ha fo } \\
\text { bispynbac }\end{array}$ & $\begin{array}{l}33.10 \mathrm{~A} \\
(5.57 \mathrm{~A})\end{array}$ & $\begin{array}{l}8.50 \mathrm{AB} \\
(2.99 \mathrm{~B})\end{array}$ & $\begin{array}{l}8.30 \mathrm{C} \\
(2.93 \mathrm{C})\end{array}$ & (3.55DE) & $\begin{array}{l}24.60 \mathrm{D} \\
(5.00 \mathrm{C})\end{array}$ & $\begin{array}{l}6.23 \mathrm{~B} \\
(2.58 \mathrm{D})\end{array}$ \\
\hline 9 & $\begin{array}{l}\text { Pendimethalin and Sesbaria co-culture } \\
\text { fb } 2,4 \mathrm{D} \text { Na salt to one hard weeding }\end{array}$ & $\begin{array}{l}45.90 \mathrm{~A} \\
(6.50 \mathrm{~A})\end{array}$ & $\begin{array}{l}11.78 \mathrm{D} \\
(3.50 \mathrm{~A})\end{array}$ & $\begin{array}{l}12.80 \mathrm{~B} \\
(3.63 \mathrm{~B})\end{array}$ & $\begin{array}{l}17.85 \mathrm{C} \\
(4.28 \mathrm{~B})\end{array}$ & $\begin{array}{l}37.30 \mathrm{~B} \\
(6.14 \mathrm{~B})\end{array}$ & $\begin{array}{l}16.85 \mathrm{C} \\
(4.16 \mathrm{~A})\end{array}$ \\
\hline \multirow[t]{5}{*}{10} & $\begin{array}{l}\text { Pendimethalin fo } 2,4 \mathrm{D} \text { fo one hand } \\
\text { weeding }\end{array}$ & $\begin{array}{c}1.80 \mathrm{~B} \\
(1.32 \mathrm{C})\end{array}$ & $\begin{array}{c}3.73 \mathrm{E} \\
(2.05 \mathrm{C})\end{array}$ & $\begin{array}{c}0.30 \mathrm{DE} \\
(0.86 \mathrm{FG})\end{array}$ & $\begin{array}{l}6.65 \mathrm{D} \\
(2.67 \mathrm{~F})\end{array}$ & $\begin{array}{c}0.90 \mathrm{~F} \\
(1.17 \mathrm{FG})\end{array}$ & $\begin{array}{l}3.13 \mathrm{D} \\
(1.89 \mathrm{E})\end{array}$ \\
\hline & Mean & $\begin{array}{l}1894 \\
(3.58)\end{array}$ & $\begin{array}{c}8.14 \\
(2.81)\end{array}$ & $5.68(2.15)$ & $\begin{array}{l}13.08 \\
(3.52)\end{array}$ & $\begin{array}{l}16.35 \\
(3.41)\end{array}$ & $\begin{array}{c}9.67 \\
(2.98)\end{array}$ \\
\hline & $\mathrm{CV} \%$ & $\begin{array}{c}60.17 \\
(2622)\end{array}$ & $\begin{array}{l}1637 \\
(7.63)\end{array}$ & $\begin{array}{c}32.79 \\
(14.51)\end{array}$ & $\begin{array}{l}14.96 \\
(7.42)\end{array}$ & $\begin{array}{l}18.50 \\
(9.65)\end{array}$ & $\begin{array}{l}14.41 \\
(6.77)\end{array}$ \\
\hline & F-test & $* *$ & $* *$ & *** & $* *$ & $* *$ & *** \\
\hline & LSD at 0.05 & 1653 & 1.934 & 2.702 & 2.837 & 4.388 & 2.023 \\
\hline
\end{tabular}

Data pertaining to weed density significantly influenced by integrated weed management practices in dry direct seeded rice on all types of weeds in both years. The weed density at 30 and 60 days after sowing differed significantly due to integrated weed management practices. The highest number of weeds was recorded in weedy check followed by bispyribac for broad leaves, sedges and grasses in 2010 and 2011 while the lowest weed population were recorded in weed free plot followed by pendimethalin, followed by $2,4-\mathrm{D}_{2}$ followed by one 
K.P. Bhurer et al./Influence of Integrated Weed.....

hand weeding. The number of weeds was comparatively less in 2011 perhaps due to more rainfall which has suppressed growth of some weeds species. This indicates that one cannot rely on herbicide only for the control of weeds and at least one hand weeding is needed. During both years, the proportion of grassy weed dry weight was higher than other weeds. Grasses persist in all of the principal crops and are a major cause for concern (Mortimer \& Richer 2001). It is also reported that the greatest weed pressure and crop-weed competition occurs in aerobic rice and least in transplanted irrigated and rain-fed lowland rice (Datta et al. 1996, Moody 1991 and Rao et al. 2007). On the other hand, weedy check had significantly the highest weed intensity and dry biomass over all the treatments.

Table 3. Dry weed weight at 30 DAS as influenced by integrated weed management practices in dry direct seeded rice at Parwanipur, Bara

\begin{tabular}{|c|c|c|c|c|c|c|c|}
\hline \multirow{3}{*}{$\mathrm{SH}$} & \multirow[b]{2}{*}{ Treatments } & \multicolumn{6}{|c|}{ Dry weed we ight $\left(\mathrm{g}^{\prime} \mathrm{m}^{2}\right) 30 \mathrm{DAS}$} \\
\hline & & \multicolumn{2}{|c|}{ Broad leaves } & \multicolumn{2}{|c|}{ Sedges } & \multicolumn{2}{|c|}{ Grasses } \\
\hline & & 2010 & 2011 & 2010 & 2011 & 2010 & 2011 \\
\hline 1 & Weedy & $19.72 \mathrm{~A}(4.34 \mathrm{a})$ & $\begin{array}{c}2.65 \mathrm{~B} \\
(1.77 \mathrm{~A})\end{array}$ & $\begin{array}{c}7.13 \mathrm{~A} \\
(2.96 \mathrm{~B})\end{array}$ & $\begin{array}{l}2.21 \mathrm{AB} \\
(1.65 \mathrm{~A})\end{array}$ & $\begin{array}{l}18.32 \mathrm{~A} \\
(4.30 \mathrm{~A})\end{array}$ & $\begin{array}{l}2.24 \mathrm{~B} \\
(1.66 \mathrm{~A})\end{array}$ \\
\hline 2 & Weed free & $0.00 \mathrm{C}(0.71 \mathrm{C})$ & $\begin{array}{c}0.00 \mathrm{~B} \\
(0.71 \mathrm{E})\end{array}$ & $\begin{array}{c}0.0 \mathrm{~B} \\
(0.71 \mathrm{~F})\end{array}$ & $\begin{array}{l}0.00 \mathrm{AB} \\
(0.71 \mathrm{~F})\end{array}$ & $\begin{array}{c}0.00 \mathrm{C} \\
(0.71 \mathrm{E})\end{array}$ & $\begin{array}{l}0.00 \mathrm{~B} \\
(0.71 \mathrm{~F})\end{array}$ \\
\hline 3 & $\begin{array}{l}\text { Pendimethalin fb } \\
\text { bispyribac }\end{array}$ & $1006 \mathrm{~B}(2.86 \mathrm{~B})$ & $\begin{array}{c}1.68 \mathrm{C} \\
(1.48 \mathrm{~B})\end{array}$ & $\begin{array}{l}21.55 \mathrm{C} \\
(4.70 \mathrm{~A})\end{array}$ & $\begin{array}{c}1.33 \mathrm{C} \\
(135 \mathrm{~B})\end{array}$ & $\begin{array}{c}9.09 \mathrm{~B} \\
(3.09 \mathrm{~B})\end{array}$ & $\begin{array}{c}1.71 \mathrm{D} \\
(1.49 \mathrm{~B})\end{array}$ \\
\hline 4 & $\begin{array}{l}\text { Pendimethalin fo two hand } \\
\text { weeding }\end{array}$ & $0.53 \mathrm{C}(1.00 \mathrm{C})$ & $\begin{array}{c}0.07 \mathrm{~B} \\
(0.75 \mathrm{DE})\end{array}$ & $\begin{array}{c}0.27 \mathrm{E} \\
(0.88 \mathrm{E})\end{array}$ & $\begin{array}{l}0.23 \mathrm{AB} \\
(0.8 \mathrm{SE})\end{array}$ & $\begin{array}{c}0.64 \mathrm{C} \\
(1.0 \pi \mathrm{DEF})\end{array}$ & $\begin{array}{c}0.41 \mathrm{~B} \\
(0.95 \mathrm{D})\end{array}$ \\
\hline 5 & Stale seedbed fb bispynbac & $1.14 \mathrm{C}(1.19 \mathrm{C})$ & $\begin{array}{c}0.13 \mathrm{~B} \\
(0.79 \mathrm{D})\end{array}$ & $\begin{array}{l}0.21 \mathrm{DE} \\
(0.84 \mathbb{E F})\end{array}$ & $\begin{array}{l}0.47 \mathrm{AB} \\
(0.99 \mathrm{D})\end{array}$ & $\begin{array}{c}1.35 \mathrm{C} \\
(1.36 \mathrm{D})\end{array}$ & $\begin{array}{l}0.61 \mathrm{~B} \\
(1.06 \mathrm{C})\end{array}$ \\
\hline 6 & $\begin{array}{l}\text { Stale seedbed fo } \\
\text { Pendimethalin fb bispynbac }\end{array}$ & $1.01 \mathrm{C}(1.48 \mathrm{C})$ & $\begin{array}{c}0.08 \mathrm{C} \\
(0.76 \mathrm{DE})\end{array}$ & $\begin{array}{c}0.09 \mathrm{D} \\
(0.7 \mathrm{TEF})\end{array}$ & $\begin{array}{r}0.06 \mathrm{C} \\
(0.74 \mathrm{~F})\end{array}$ & $\begin{array}{c}0.81 \mathrm{C} \\
(1.15 \mathrm{DE})\end{array}$ & $\begin{array}{c}0.33 \mathrm{E} \\
(0.91 \mathrm{DE})\end{array}$ \\
\hline 7 & $\begin{array}{l}\text { Mhylch 4t/ha fb bispynbac fo } \\
\text { One hand weeding }\end{array}$ & $1.92 \mathrm{C}(1.48 \mathrm{C})$ & $\begin{array}{c}1.18 \mathrm{C} \\
(1.30 \mathrm{C})\end{array}$ & $\begin{array}{l}1.00 \mathrm{DE} \\
(122 \mathrm{D})\end{array}$ & $\begin{array}{c}0.81 \mathrm{C} \\
(1.14 \mathrm{C})\end{array}$ & $\begin{array}{l}2.76 \mathrm{C} \\
(1.80 \mathrm{C})\end{array}$ & $\begin{array}{l}1.51 \mathrm{E} \\
(1.41 \mathrm{~B})\end{array}$ \\
\hline 8 & $\begin{array}{l}\text { Stale seedbed fo mulch4tha } \\
\text { fo bispynbac }\end{array}$ & $9.98 \mathrm{~B}(1.20 \mathrm{~B})$ & $\begin{array}{c}0.14 \mathrm{C} \\
(0.80 \mathrm{D})\end{array}$ & $\begin{array}{c}2.39 \mathrm{E} \\
(1.70 \mathrm{C})\end{array}$ & $\begin{array}{c}0.29 \mathrm{C} \\
(0.89 \mathrm{E})\end{array}$ & $\begin{array}{c}9.32 \mathrm{~B} \\
(3.12 \mathrm{~B})\end{array}$ & $\begin{array}{l}0.64 C D \\
(1.0 \pi)\end{array}$ \\
\hline 9 & $\begin{array}{l}\text { Pendimethalin ard Sesbania } \\
\text { co-culture fo } 2,4 \mathrm{D} \text { Na salt } \\
\text { fo one hard weeding }\end{array}$ & $8.83 \mathrm{~B}(0.95 \mathrm{~B})$ & $\begin{array}{c}1.54 \mathrm{~A} \\
(1.42 \mathrm{~B})\end{array}$ & $\begin{array}{c}2.13 \mathrm{E} \\
(1.62 \mathrm{C})\end{array}$ & $\begin{array}{c}1.13 \mathrm{~A} \\
(127 \mathrm{~B})\end{array}$ & $\begin{array}{c}9.04 \mathrm{~B} \\
(3.08 \mathrm{~B})\end{array}$ & $\begin{array}{c}1.64 \mathrm{~A} \\
(1.46 \mathrm{~B})\end{array}$ \\
\hline \multirow[t]{5}{*}{10} & $\begin{array}{l}\text { Pendimethalin fb } 2,4 \mathrm{D} \text { fo } \\
\text { one hand weeding }\end{array}$ & $0.44 \mathrm{C}(3.07 \mathrm{C})$ & $\begin{array}{c}0.03 \mathrm{C} \\
(0.73 \mathrm{DE})\end{array}$ & $\begin{array}{c}0.08 \mathrm{C} \\
(0.76 \mathrm{EF})\end{array}$ & $\begin{array}{c}0.03 \mathrm{~B} \\
(0.73 \mathrm{~F})\end{array}$ & $\begin{array}{c}0.29 \mathrm{C} \\
(0.89 \mathrm{EF})\end{array}$ & $\begin{array}{c}0.20 \mathrm{C} \\
(0.84 \mathrm{E})\end{array}$ \\
\hline & Mean & $5.36(1.99)$ & $\begin{array}{c}0.75 \\
(1.05)\end{array}$ & $3.48(1.59)$ & $0.65(1.03)$ & $5.16(206)$ & $0.93(1.15)$ \\
\hline & $\mathrm{CV} \%$ & $70.25(26.65)$ & $\begin{array}{l}17.78 \\
(4.22)\end{array}$ & $\begin{array}{l}15.71 \\
(5.80)\end{array}$ & $\begin{array}{l}23.05 \\
(5.79)\end{array}$ & $\begin{array}{c}40.28 \\
(12.28)\end{array}$ & $\begin{array}{l}17.63 \\
(5.19)\end{array}$ \\
\hline & F-test & $* * *$ & $* * k$ & $* *$ & $* *$ & $* *$ & $* *$ \\
\hline & LSD at 0.05 & 5.465 & 0.1947 & 0.7827 & 0.22 & 30.16 & 0.2384 \\
\hline
\end{tabular}

\section{Weed control efficiency}

Mean data concerning to the effect of the various methods of weeds control verses weedy check on weed control efficiency and weed index showed highly significant difference among them at different growth periods i.e. at 30 and 60 DAS during 2010 and 2011 which have been presented in Table-5. Higher weed control efficiencies resulted in pendimethalin followed by 2, 4- D followed by one hand weeding and pendimethalin followed by two hand weeding and they were at par with weedy check. Experimental site got continuous rainfall in the year 2011, thus, less number of weeds were observed . On the other hand, low weed index resulted in those treatments. Statistically similar weed control efficiencie-were obtained with stale seed bed followed by pendimethalin followed bispyribac. However, weed control in each case was decreased in 2011 as compared to 2010 which might be due to more rainfall favoring growth of plant while the lowest weed index was observed in pendimathalin, followed by 2,4-D followed by one hand weeding and it was immediately followed by treatment 
pendimethalin followed by two hand weeding and stale seed bed followed by pendimethalin followed by bispyribac. In each case, the involvement of pendimethalin showed significantly role in controlling weeds and more effectiveness this pre-emergence herbicide in Dry DSR on the basis result. -No doubt weed free check had the highest weed control efficiency and weedy check had the highest weed index.

Table 4. Dry weed weight at 60 DAS as influenced by integrated weed management practices in dry direct seeded rice at Parwanipur, Bara

\begin{tabular}{|c|c|c|c|c|c|c|c|}
\hline \multirow[b]{2}{*}{ SN } & \multirow[b]{2}{*}{ Treatments } & \multicolumn{6}{|c|}{ Dry weed weight $\left(\mathrm{g}^{2} \mathrm{~m}^{2} 60 \mathrm{DAS}\right.$} \\
\hline & & \multicolumn{2}{|c|}{ Broad leaves } & \multicolumn{2}{|c|}{ Sedges } & \multicolumn{2}{|c|}{ Grasses } \\
\hline \multirow{3}{*}{1} & & 2010 & 2011 & 2010 & 2011 & 2010 & 2011 \\
\hline & Weedy & $42.46 \mathrm{~A}$ & $11.28 \mathrm{~B}$ & $15.43 \mathrm{~A}$ & $20.22 \mathrm{~B}$ & $47.53 \mathrm{~A}$ & $1758 \mathrm{~A}$ \\
\hline & & $(6.45 \mathrm{~A})$ & (3.43A) & $(3.97 \mathrm{~A})$ & $(4.55 \mathrm{~A})$ & $(6.92 \mathrm{~A})$ & $(4.25 \mathrm{~A})$ \\
\hline 2 & Weed free & $0.00 \mathrm{~B}$ & $0.00 \mathrm{~B}$ & $0.00 \mathrm{D}$ & $0.00 \mathrm{~B}$ & $0.00 \mathrm{E}$ & $0.00 \mathrm{~A}(0.71 \mathrm{~F})$ \\
\hline \multirow{3}{*}{3} & & $(0.71 C)$ & $(0.71 \mathrm{~F})$ & $(0.71 G)$ & $(0.71 \mathrm{G})$ & $(0.71 G)$ & \\
\hline & Pencimethalin fo bispynbac & $46.13 \mathrm{~A}$ & $11.56 \mathrm{~B}$ & $12.63 \mathrm{AB}$ & $17.22 \mathrm{AB}$ & $34.23 \mathrm{~B}$ & $1722 \mathrm{~A}$ \\
\hline & 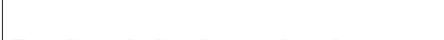 & $(6.78 \mathrm{~A})$ & $(3.46 \mathrm{~A})$ & $(3.61 \mathrm{AB})$ & $(4.20 \mathrm{AB})$ & $(5.88 \mathrm{~B})$ & $(4.20 \mathrm{~A})$ \\
\hline \multirow{2}{*}{4} & Pencimethalin fo two hand & $3.02 \mathrm{~B}$ & $5.44 \mathrm{AB}$ & $0.86 \mathrm{D}$ & $10.67 \mathrm{AB}$ & $1.20 \mathrm{E}$ & $3.83 \mathrm{~A}$ \\
\hline & weeding & $(1.75 \mathrm{BC})$ & (2.43D) & $(1.14 \mathrm{EFG})$ & (3.33E) & $(1.30 \mathrm{~F})$ & (2.08E) \\
\hline \multirow{2}{*}{5} & Stale seedbed fb bispynbac & $4.10 \mathrm{~B}$ & $10.91 \mathrm{~B}$ & $1.94 \mathrm{D}$ & $16.49 \mathrm{~B}$ & $4.96 \mathrm{DE}$ & $10.70 \mathrm{~A}$ \\
\hline & & $(2.00 \mathrm{BC})$ & $(3.37 \mathrm{AB})$ & $(1.56 \mathrm{DE})$ & $(4.11 \mathrm{BC})$ & $(2.30 \mathrm{E})$ & (3.34C) \\
\hline \multirow{2}{*}{6} & Stale seedbed fo Pendimethalin to & $3.24 \mathrm{~B}$ & $8.98 \mathrm{~B}$ & $1.15 \mathrm{D}$ & $14.82 \mathrm{~B}$ & $1.98 \mathrm{E}$ & $9.63 \mathrm{~A}$ \\
\hline & bispynbac & $(1.84 \mathrm{BC})$ & $(3.07 \mathrm{BC})$ & $(1.28 \mathrm{DEF})$ & (3.91BCD) & $(1.55 \mathrm{~F})$ & $(3.18 \mathrm{C})$ \\
\hline \multirow{2}{*}{7} & Mulch 4t/ha fo bispynbac to One & $6.64 \mathrm{~B}$ & $8.63 \mathrm{D}$ & $2.57 \mathrm{D}$ & $1358 \mathrm{D}$ & $7.72 \mathrm{D}$ & $10.29 \mathrm{C}$ \\
\hline & hand weeding & (2.61B) & $(3.02 \mathrm{C})$ & $(1.73 \mathrm{D})$ & (3.74CD) & $(2.85 \mathrm{D})$ & $(3.28 \mathrm{C})$ \\
\hline \multirow{3}{*}{8} & Stale seedbed fo mulch4t/ha fo & $33.91 \mathrm{~A}$ & $8.71 \mathrm{~A}$ & $8.50 \mathrm{C}$ & $12.47 \mathrm{~A})$ & $25.20 \mathrm{C}$ & $6.38 \mathrm{~A}$ \\
\hline & bispynbac & $(5.63 \mathrm{~A})$ & $(3.03 \mathrm{C})$ & $(2.97 \mathrm{C})$ & $(3.59 \mathrm{DE})$ & $(5.06 \mathrm{C})$ & (2.61E) \\
\hline & Pendimethalin and Sesbaria co- & $38.08 \mathrm{~A}$ & $9.77 \mathrm{C}$ & $10.62 \mathrm{BC}$ & $14.81 \mathrm{AC}$ & $30.94 \mathrm{~B}$ & $13.98 \mathrm{~B}$ \\
\hline \multirow[t]{2}{*}{9} & $\begin{array}{l}\text { culture fb } 2,4 \text { D Na salt fb one hard } \\
\text { weeding }\end{array}$ & $(5.93 \mathrm{~A})$ & $\left(\begin{array}{c}3.20 \mathrm{ABC} \\
)\end{array}\right.$ & $(3.32 \mathrm{BC})$ & (3.91BCD) & $(2.60 \mathrm{~B})$ & $(3.80 \mathrm{~B})$ \\
\hline & Pencimethalin fo $2,4 \mathrm{D}$ fo one & $1.62 \mathrm{~B}$ & $3.34 \mathrm{D}$ & $0.27 \mathrm{D}$ & $5.96 \mathrm{D}$ & $0.81 \mathrm{E}$ & $2.80 \mathrm{C}(1.81 \mathrm{E})$ \\
\hline \multirow[t]{5}{*}{10} & hand weeding & $(1.28 \mathrm{BC})$ & $(1.96 \mathrm{E})$ & $(0.85 \mathrm{FG})$ & $(2.54 \mathrm{~F})$ & $(1.14 \mathrm{FG}$ & \\
\hline & Mean & $\begin{array}{l}17.92 \\
(3.51)\end{array}$ & $7.86(2.77)$ & $5.40(2.11)$ & $\begin{array}{l}12.62 \\
(3.46)\end{array}$ & $\begin{array}{l}15.46 \\
(3.33)\end{array}$ & $9.24(292)$ \\
\hline & $\mathrm{CV} \%$ & $\begin{array}{l}56.05 \\
(25.20)\end{array}$ & $\begin{array}{l}17.31 \\
0.82)\end{array}$ & $\begin{array}{c}35.95 \\
(15.12)\end{array}$ & $\begin{array}{l}15.72 \\
(7.67)\end{array}$ & $\begin{array}{l}22.79 \\
(10.59)\end{array}$ & $15.98(7.20)$ \\
\hline & F-test & $* *$ & **t & $* *$ & $* *$ & ***: & $* * *$ \\
\hline & LSD at 005 & 14.57 & 1.974 & 2.814 & 2.88 & 5.11 & 2.142 \\
\hline
\end{tabular}

\section{Grain and straw yield}

Grain yield, straw yield, grain straw ratio and harvest index as influenced by integrated weed management practices are presented in Table-6. Observation of grain yield, straw yield, ration grain and straw ratio, and harvest index recorded significantly different among tested treatments due to integrated weed management practices during both years. The highest grain and straw yield obtained in weed free check closely followed by pendimethalin followed by 2, 4- D followed by one hand weeding, pendimethalin followed by two hand weeding and stale seed bed followed by pendimethalin, followed by Bispyribac and these were statistically at par with each other. Overall, the involvement of pendimethalin followed by manual weeding or other herbicides indicates that pendimethalin seems to be an effective pre-emergence herbicide for weed control in direct seeded rice. The efficacy of Pendimethalin alone (Moody 1991 and Valverde et al. 2005) or in combination with hand weeding in controlling weeds in dry direct seeded rice (Dry DSR) was reported highly responsive for controlling weeds (Ramamoorthy et al. 1998 and Singh et al. 2005). Overall, the grain and straw yield were lower in 2010 than 2011 because of erratic rainfall in occurrence of drought especially during active crop growth stage. However, rainfall pattern of monsoon in the second year was well distributed during crop growth stages of rice resulting in better crop performance. The experimental plots were also heavily infested with gundhi bug (L. varicornis) which subsequently reduced yield in 2010 . 
K. P. Bhurer et al./Influence of Integrated Weed.....

Table 5. Weed control efficiency and weed index as influenced by different weed management treatments at RARS, Parwanipur 2010/11

\begin{tabular}{|c|c|c|c|c|c|c|c|}
\hline \multirow[b]{2}{*}{$\mathrm{SH}$} & \multirow[b]{2}{*}{ Treatments } & \multicolumn{4}{|c|}{ Weedcontrol efficiency } & \multirow{2}{*}{\multicolumn{2}{|c|}{ Weed Index }} \\
\hline & & \multicolumn{2}{|c|}{$30 \mathrm{DAS}$} & \multicolumn{2}{|c|}{$60 \mathrm{DAS}$} & & \\
\hline & & 2010 & 2011 & 2010 & 2011 & 2010 & 2011 \\
\hline 1 & Weedy & $0.00 \mathrm{E}$ & $16.63 \mathrm{D}$ & $0.00 \mathrm{E}$ & $0.00 \mathrm{C}$ & $89.54 \mathrm{~A}$ & $67.41 \mathrm{BC}$ \\
\hline 2 & Weed free & $100.00 \mathrm{~A}$ & $100.00 \mathrm{D}$ & $100.00 \mathrm{~A}$ & $100.00 \mathrm{D}$ & $0.00 \mathrm{E}$ & $0.00 \mathrm{~A}$ \\
\hline 3 & Pendimethalin fo bispynbac & $43.93 \mathrm{C}$ & $3623 \mathrm{BBC}$ & $21.46 \mathrm{D}$ & $16.79 \mathrm{BC}$ & $24.01 \mathrm{BC}$ & $17.51 \mathrm{D}$ \\
\hline 4 & Pencimethalin to two hand weeding & $94.86 \mathrm{~A}$ & $91.20 \mathrm{~A}$ & $95.99 \mathrm{~A}$ & $65.05 \mathrm{~A}$ & $6.23 \mathrm{DE}$ & $4.47 \mathrm{E}$ \\
\hline 5 & Stale seedbed fb bispynbac & $90.31 \mathrm{AB}$ & $82.04 \mathrm{C}$ & $90.83 \mathrm{AB}$ & $39.42 \mathrm{BC}$ & $13.22 \mathrm{CD}$ & $7.46 \mathrm{~B}$ \\
\hline 6 & $\begin{array}{l}\text { Stale seedbed fo Pendimethalin fb } \\
\text { bispynbac }\end{array}$ & $92.55 \mathrm{AB}$ & $87.44 \mathrm{E}$ & $94.70 \mathrm{~A}$ & $49.01 \mathrm{D}$ & $13.24 \mathrm{CD}$ & $4.05 \mathrm{E}$ \\
\hline 7 & $\begin{array}{l}\text { Mulch 4t/ha fo bispyibac fo One hand } \\
\text { weeding }\end{array}$ & $84.47 \mathrm{~B}$ & $76.00 \mathrm{C}$ & $82.28 \mathrm{~B}$ & $33.29 \mathrm{BC}$ & $15.09 \mathrm{CD}$ & $8.05 \mathrm{BC}$ \\
\hline 8 & $\begin{array}{l}\text { Stale seedbed fo roulch4tha fb } \\
\text { bispynbac }\end{array}$ & $15.35 \mathrm{D}$ & $33.11 \mathrm{~B}$ & $22.49 \mathrm{D}$ & $11.84 \mathrm{BC}$ & $32.47 \mathrm{~B}$ & $25.63 \mathrm{D}$ \\
\hline 9 & $\begin{array}{l}\text { Pencimethalin and Sesbaria co-culture } \\
\text { fb2,4D Na salt fb one hand weeding }\end{array}$ & $52.66 \mathrm{C}$ & $5122 \mathrm{BC}$ & $42.63 \mathrm{C}$ & $25.98 \mathrm{BC}$ & $7.63 \mathrm{DE}$ & $10.63 \mathrm{~B}$ \\
\hline \multirow[t]{5}{*}{10} & $\begin{array}{l}\text { Pendimethalin fo } 2,4 \mathrm{D} \text { fo one hand } \\
\text { weeding }\end{array}$ & $95.91 \mathrm{~A}$ & $9468 \mathrm{BC}$ & $97.40 \mathrm{~A}$ & $74.40 \mathrm{~B}$ & $3.99 \mathrm{DE}$ & $2.36 \mathrm{CD}$ \\
\hline & Mean & 67 & $66.85 \mathrm{~A}$ & 64.78 & 41.58 & 20.54 & 14.76 \\
\hline & $\mathrm{CV} \%$ & 9.54 & 9.79 & 12.08 & 16.53 & 38.25 & 21.22 \\
\hline & F-test & $* *$ & $* *$ & $* *$ & *** & $* *$ & $* *$ \\
\hline & LSD at 0.05 & 9.277 & 13.25 & 11.35 & 9.97 & 11.4 & 4.544 \\
\hline
\end{tabular}

Table 6. Grain yield, straw yield, grain straw ratio and harvest index as influenced by different weed management treatments at RARS, Parwanipur during 2010 and 2011

\begin{tabular}{|c|c|c|c|c|c|c|c|c|c|}
\hline \multirow{3}{*}{$\mathrm{SH}$} & \multirow{3}{*}{ Treatments } & \multirow{2}{*}{\multicolumn{2}{|c|}{ Grain Yield(kg/ha) }} & \multirow{2}{*}{\multicolumn{2}{|c|}{ Straw Yield (kg/ha) }} & \multirow{2}{*}{\multicolumn{2}{|c|}{ Grain : Straw }} & \multirow{2}{*}{\multicolumn{2}{|c|}{ Harvest irdex }} \\
\hline & & & & & & & & & \\
\hline & & 2010 & 2011 & 2010 & 2011 & 2010 & 2011 & 2010 & 2011 \\
\hline 1 & Weedy & $537 \mathrm{~F}$ & $2065 \mathrm{G}$ & $937 \mathrm{E}$ & $63605 \mathrm{E}$ & $0.57 \mathrm{~B}$ & $0.32 \mathrm{D}$ & $36 \mathrm{D}$ & $32 \mathrm{D}$ \\
\hline 2 & Weed free & 5305A & $6369 \mathrm{~A}$ & $6525 \mathrm{~A}$ & $9510 \mathrm{~A}$ & $0.81 \mathrm{~A}$ & $0.67 \mathrm{~A}$ & $45 \mathrm{~A}$ & $40 \mathrm{~A}$ \\
\hline 3 & $\begin{array}{l}\text { Fendimethalin fb } \\
\text { bispynbac } \\
\text { Pendimethalin fh }\end{array}$ & $3938 \mathrm{DE}$ & $5232 \mathrm{E}$ & $5147 \mathrm{C}$ & $8520 \mathrm{CD}$ & $0.76 \mathrm{~A}$ & $0.61 \mathrm{BC}$ & $43 \mathrm{~A}$ & $38 \mathrm{BC}$ \\
\hline 4 & $\begin{array}{l}\text { Fendimethalin to two } \\
\text { hand weeding }\end{array}$ & $4997 \mathrm{ABC}$ & $6351 \mathrm{~A}$ & $6178 \mathrm{~A}$ & $9157 \mathrm{AB}$ & $0.81 \mathrm{~A}$ & $0.69 \mathrm{~A}$ & $45 \mathrm{~A}$ & $41 \mathrm{~A}$ \\
\hline 5 & $\begin{array}{l}\text { Stale seedbed fb } \\
\text { bispynbac }\end{array}$ & $4525 \mathrm{BCD}$ & $5871 \mathrm{BCD}$ & $5664 \mathrm{~B}$ & $883 \mathrm{BC}$ & $0.80 \mathrm{~A}$ & $0.66 \mathrm{AB}$ & $44 \mathrm{~A}$ & $40 \mathrm{~A}$ \\
\hline 6 & $\begin{array}{l}\text { Stale seedbed fb } \\
\text { Pendimethalin fo } \\
\text { bispynbac }\end{array}$ & $4971 \mathrm{ABC}$ & $6134 \mathrm{ABC}$ & $6164 \mathrm{~A}$ & $8935 \mathrm{ABC}$ & $0.81 \mathrm{~A}$ & $0.69 \mathrm{~A}$ & $45 \mathrm{~A}$ & $41 \mathrm{~A}$ \\
\hline 7 & fo One hand weeding & $4513 \mathrm{BCD}$ & $5883 \mathrm{CD}$ & $5660 \mathrm{~B}$ & $8817 \mathrm{BC}$ & $0.80 \mathrm{~A}$ & $0.66 \mathrm{~A}$ & $44 \mathrm{~A}$ & $40 \mathrm{~A}$ \\
\hline
\end{tabular}


Nepal Journal of Science and Technology Vol. 14, No. 1 (2013) 33-42

\begin{tabular}{|c|c|c|c|c|c|c|c|c|c|}
\hline 8 & $\begin{array}{l}\text { Stale seedbed fo } \\
\text { mulch4t/ha fo bispynbac }\end{array}$ & $3485 \mathrm{E}$ & $4719 \mathrm{~F}$ & $4000 \mathrm{D}$ & $8146 \mathrm{D}$ & $0.87 \mathrm{~A}$ & $0.58 \mathrm{C}$ & $4 \pi \mathrm{B}$ & $37 \mathrm{C}$ \\
\hline 9 & $\begin{array}{l}\text { Pendimethalin and } \\
\text { Sesoania co-culture fo } 2,4 \\
\text { D Na salt to one hand } \\
\text { weeding }\end{array}$ & $4385 \mathrm{CD}$ & $5671 \mathrm{D}$ & $5699 \mathrm{~B}$ & $8742 \mathrm{BC}$ & $0.77 \mathrm{~A}$ & $0.65 \mathrm{AB}$ & $44 \mathrm{~A}$ & $39 \mathrm{AB}$ \\
\hline \multirow[t]{5}{*}{10} & $\begin{array}{l}\text { Pendimethalin fo } 2,4 \mathrm{D} \text { fb } \\
\text { one hard weeding }\end{array}$ & $5161 \mathrm{AB}$ & $6160 \mathrm{AB}$ & $6268 \mathrm{~A}$ & $9114 \mathrm{AB}$ & $0.82 \mathrm{~A}$ & $068 \mathrm{~A}$ & $45 \mathrm{~A}$ & $40 \mathrm{~A}$ \\
\hline & Mean & 4181.65 & 544055 & 5220 & 8618.25 & 0.78 & 0.62 & 43.69 & 38.05 \\
\hline & $\mathrm{CV} \%$ & 10.86 & 3.66 & 6.17 & 4.41 & 10.57 & 6.07 & 6.34 & 3.76 \\
\hline & F-test & $* * *$ & $* *$ & $* *$ & $* * *$ & **t & $* *$ & $* *$ & $* *$ \\
\hline & LSD at 0.05 & 699 & 290.2 & 4675 & 554.9 & 0.121 & 0.05 & 4.02 & 2.00 \\
\hline
\end{tabular}

Table 7. Economic analysis as influenced by integrated weed management practices in dry direct seeded rice at

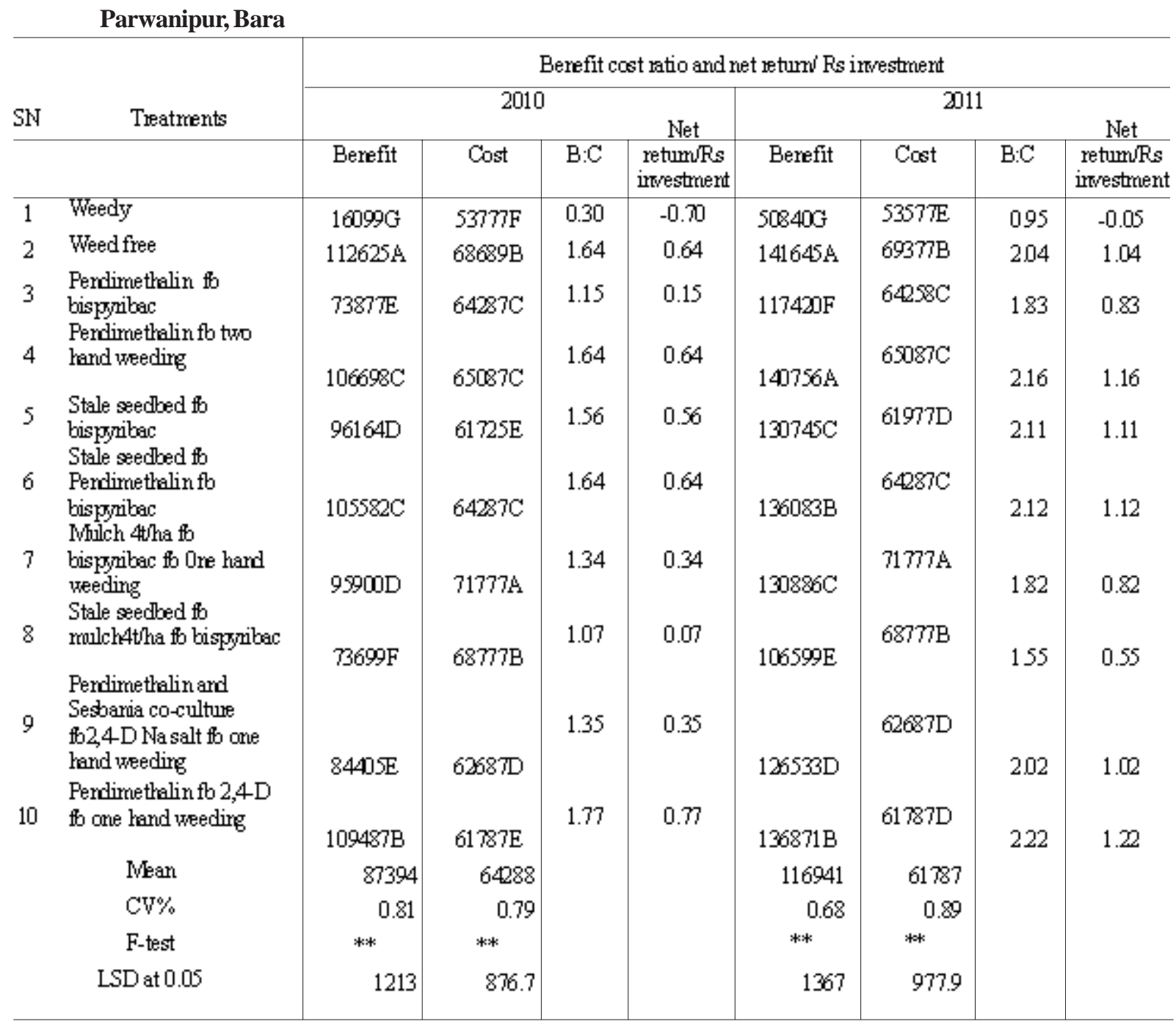


K. P. Bhurer et al./Influence of Integrated Weed.....

\section{Economics}

A perusal of data on economic analysis of integrated weed management practices in dry direct seeded rice suggested that cost of cultivation was almost the same in both years, but net returns were almost double in the year 2011 than that in 2010 (Table-6) due to more grain and straw_production. The highest yield resulted in weed free check followed by pendimethalin followed by two hand weeding and pendimethalin, followed by 2, 4- D followed by one hand weeding. However, the benefit cost ratio and net return per unit investment resulted the highest in pendimethalin followed by 2, 4$\mathrm{D}$ followed by one hand weeding due to saving of labour cost.

Besides the net return per unit investment exceed by stale seed bed followed by bispyribac, and stale seed bed followed by pendimethalin, followed by bispyribac as compared to weed free check. This revealed that amid increasing wage rate and labour scarcity, integrated weed management through pendimethalin 30 EC@1 kg a.i. /ha as pre- emergence herbicide application followed by 2, 4- D sodium salt (80 WP) @ $0.5 \mathrm{~kg}$ a.i. /ha followed by one hand weeding or stale seed bed followed by pendimethalin (30 EC) @1 kg a.i. /ha followed by bispyribac (Nominee gold 10\%) @25 g a.i. /ha 10\% @ 200ml/ha at 20 days of seeding resulted best alternative for manual hand weeding practices giving higher net return per unit investment.

\section{References}

Behera, A.K. and S.N. Jena. 1998. Weed control in direct seeded, rain-fed up land rice (Oryza sativa). Indian Journal of Agronomy 43(2): 284-290.

De Datta, S.K. and A.M. Beltazar. 1996. Weed control technology as a component of rice production systems. In: Weed management in rice. FAO Plant Production and Protection Division. (Eds. B.A. Auld; K.U. Kim). paper 139,FAO, Rome. pp 27-52.

Kumar, V and J.K. Ladha. 2011. Direct seeded of rice. Recent developments and future research needs. Advances in Agronomy 111:299-413.
MOAC, 2011/012. Statistical information Nepalese agriculture. Agri-Business, Promotion and Statistics Division, Ministry of Agriculture.

Moody, K. 1991. Weed management in rice. In: Handbook of pest management in agriculture, (Ed. D. Pimental). $2^{\text {nd }}$ Ed. CRC Press, Boca Raton. FL. USA. pp 301328.

Ramamoorthy, K., A. Arokiaraj and A. Balasubramanian. 1998. Effect of irrigation and chemical weed control on crop yield and nutrient uptake by upland rice and associated weeds under rice-blackgram intercropping system. Oryza 33: 264-268

Ranjit, J.D. 2007. Weeds associated with different crops. Paper presented to Training on Weed management in rice April 25-27, 2007, NARC, Khumaltar.

Rao, A.N., D.E. Johnsson, B. Siva Prasad, J.K. Ladha and A.M. Mortimer. 2007. Weed management in direct seeded rice. Adv. Agron.93: 153-255.

Rodder, W. 2001. Slash and burn rice systems in the hills of Northern Lao PDR. In: Description, challenges and opportunities IRRI, LOS Banos, The Philippines. pp. 201.

Sanchez, P.A. 1973. Puddling tropical soils. Effects on water losses. Soil Sci. 115: 303-308.

Singh, S., L. Bhusan, J.K. Ladha R.K. Gupta, A.N. Rao and B. Sivprasad. 2005. Weed management in dry seeded rice (Oryza sativa) cultivated in the furrow-irrigated raised-bed planting system. www. elsevier.com/locate/ cropo.

Singh, S., R.K. Sharma, R.K. Gupta and S.S. Singh. 2008. Changes in rice-wheat production technologies and how rice -wheat became a success story: Lessons from Zero-tillage wheat. In: Direct-seeding of rice and weed management in theiintegrated rice-wheat croppings System of the indo-gangetic plains. (Eds. Y.Singh, V.P.Singh, B.Chauhan, A.Orr, A.M.Mortimer, D.E. Johnson, and B.Hardy). IRRI, Philippines and Directorate of Experiment Station, G.B. P. U. A. and T. Pantnagar, India.pp.91-106.

Singh, S, L. Bhusan, J.K. Ladha, R.K. Gupta, A.N. Rao and B. Sivaprasad. 2006. Weed management in dry-seeded rice (Oryza sativa) cultivated on furrow irrigated raised bed planting System. Crop Prot. 25: 487 - 495.

Valverde, B.E. and J. Gressel. 2005. Implication and containment of gene flow from herbicide-resistant rice (Oryza sativa). In: Proceeding of $20^{\text {th }}$ Asian Pacific weed sciences society. Ho Chi Minh City, Vietnan. pp 63-84. 
Nepal Journal of Science and Technology Vol. 14, No. 2 (2013) 33-42 